\title{
Evaluating the Performance of Multiple Coulomb Scattering-Based Momentum Reconstruction with MicroBooNE Data
}

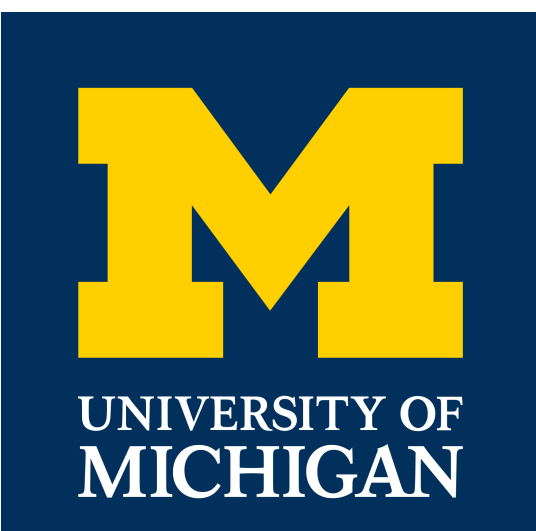

\author{
Polina Abratenko \\ University of Michigan-Ann Arbor \\ New Perspectives '18
}

This document was prepared by MicroBooNE collaboration using the resources of the Fermi National Accelerator Laboratory (Fermilab), a U.S. Department of Energy, Office of Science, HEP User Facility. Fermilab is managed by Fermi Research Alliance, LLC (FRA), acting under Contract No. DEAC02-07CH11359.

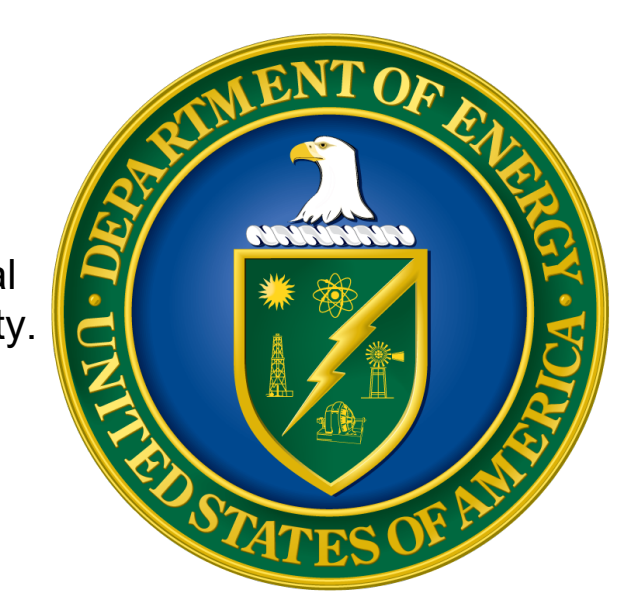




\section{The MicroBooNE Experiment}

- Goals: investigate the excess of low energy events seen by LSND/MiniBooNE (maybe due to oscillations), study neutrinoargon cross-sections, LArTPC R\&D...

- Neutrino oscillation for the two neutrino case:

$$
P_{\alpha \rightarrow \beta}=\sin ^{2}(2 \theta) \sin ^{2}\left(\frac{\Delta m^{2} L}{4(E)}\right)
$$

$\mathrm{P}=$ probability of a neutrino of flavor a later being measured to have flavor $\mathrm{B}$

$\theta=$ mixing angle

$\Delta \mathrm{m}^{2}=$ neutrino mass squared difference

$L=$ distance from neutrino source to detector

$\mathrm{E}=$ energy of neutrino 


\section{The MicroBooNE Experiment}

- For $v_{\mu}$ CC events (used in cross-section/oscillation measurements), neutrino-induced muons are used to reconstruct neutrino energy

- However, in MicroBooNE, 50\% of the time, these muon tracks are not fully contained in the TPC!

- It's not possible to use range or calorimetric methods to compute momentum; we must use Multiple Coulomb Scattering (MCS)

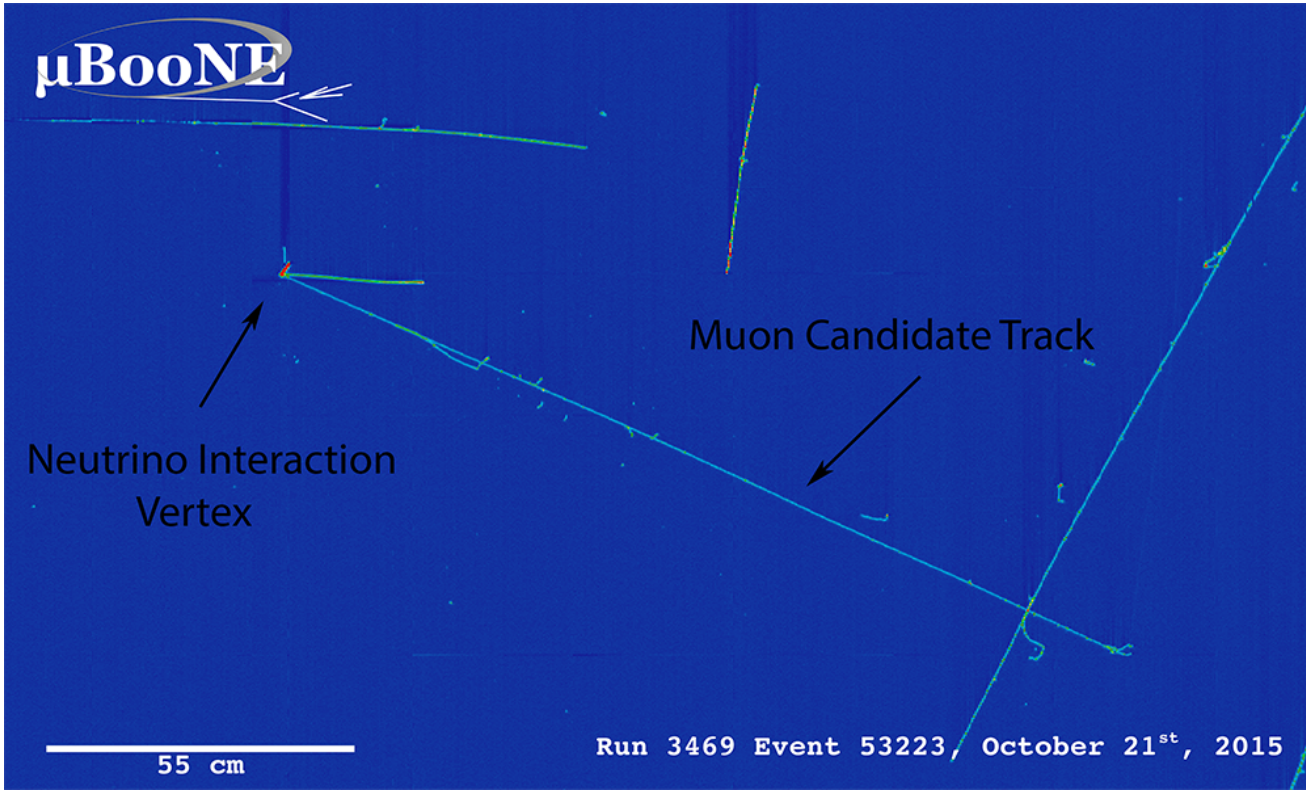




\section{Multiple Coulomb Scattering}

- When a charged particle passes through some material, it undergoes EM collisions with atomic nuclei

- After each collision, the particle's trajectory is deflected from its initial direction
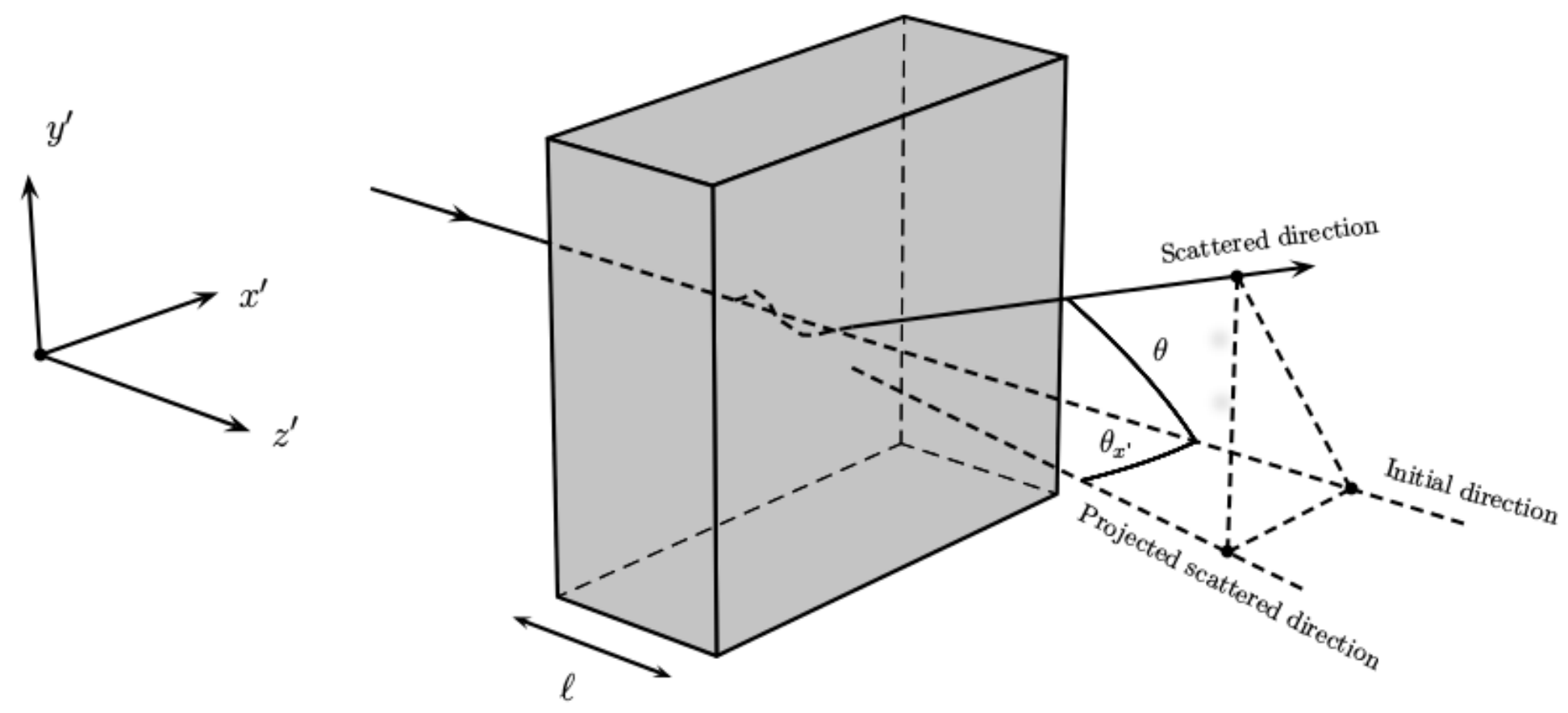


\section{Multiple Coulomb Scattering}

- The collection of these small deflections is distributed like a Gaussian, with a mean at 0 and RMS given by the tuned Highland formula:

$$
\sigma_{o}^{\mathrm{HL}}=\frac{S_{2}}{p \beta c} z \sqrt{\frac{l}{X_{0}}}\left[1+\epsilon \times \ln \left(\frac{l}{X_{0}}\right)\right]
$$

$p=$ particle momentum

$\beta=$ ratio of particle velocity to $c$,

$I=$ distance travelled inside medium
$X_{0}=$ radiation length of argon

$z=$ magnitude of charge of particle

$S_{2}, \epsilon=$ fit parameters

- We can determine the momentum of the particle if we know the angular deflections

MCS is the only way to reconstruct the energy of exiting muon tracks in MicroBooNE! 


\section{Multiple Coulomb Scattering Algorithm Overview}

- We determine these angular deflections by splitting the particle's track into segments and then computing the angle between adjacent segments

- We use the Maximum Likelihood Method to calculate the momentum of a given muon

- Input angular deflections

- Raster likelihood scan from $1 \mathrm{MeV}$ to $7.5 \mathrm{GeV}$

- Momentum and RMS updated through use of energy-range relation

- Conditions:

- Nominal segment length of $14 \mathrm{~cm}$

- Tracks must be above $100 \mathrm{~cm}$ in length 
MCS Performance on Contained Data Tracks 


\section{MCS Performance on Contained Data Tracks}

- Beam neutrino induced NumuCC data

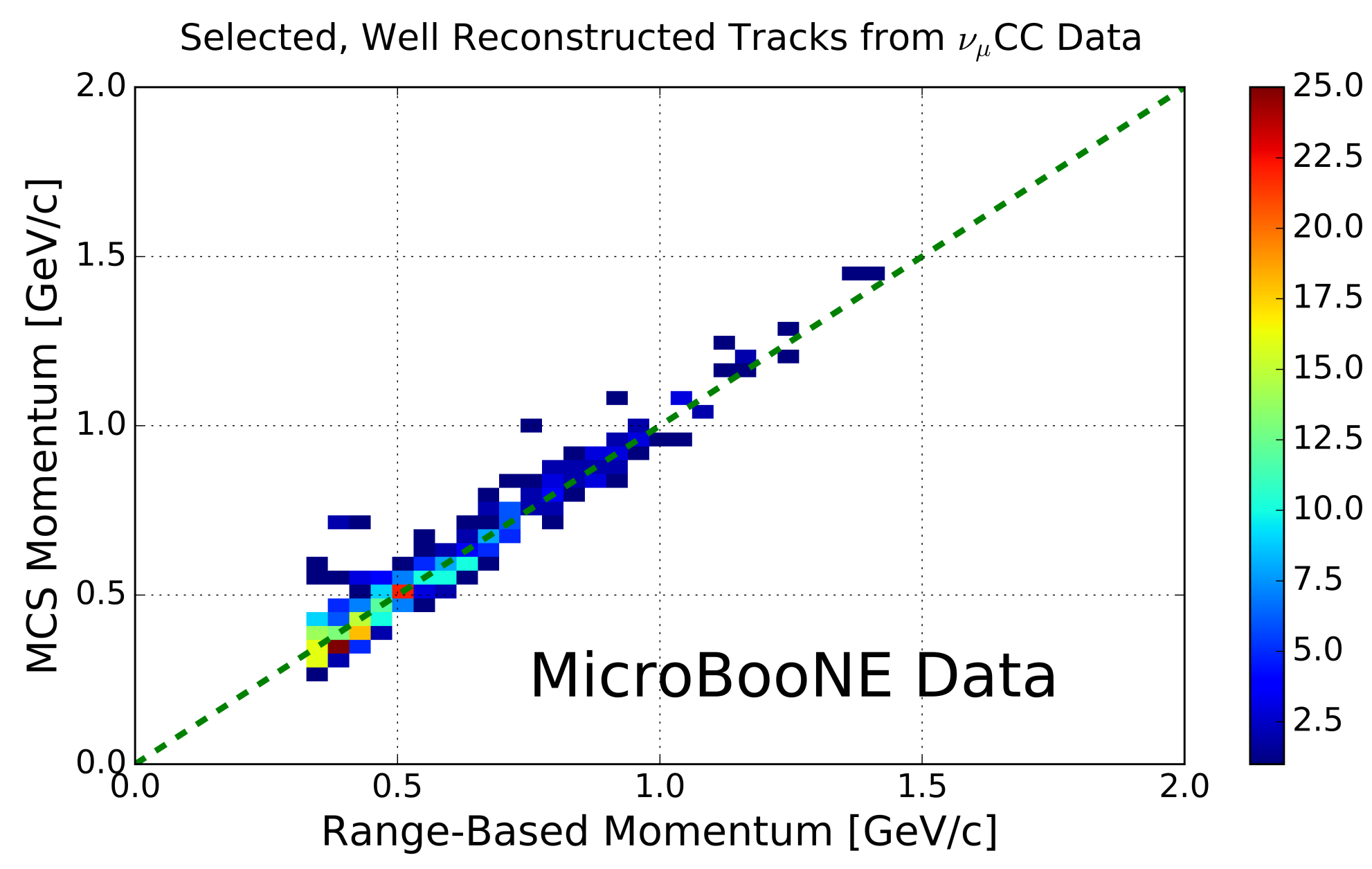




\section{MCS Performance on Contained Data Tracks}

- MCS bias vs. range momentum for both simulation and data

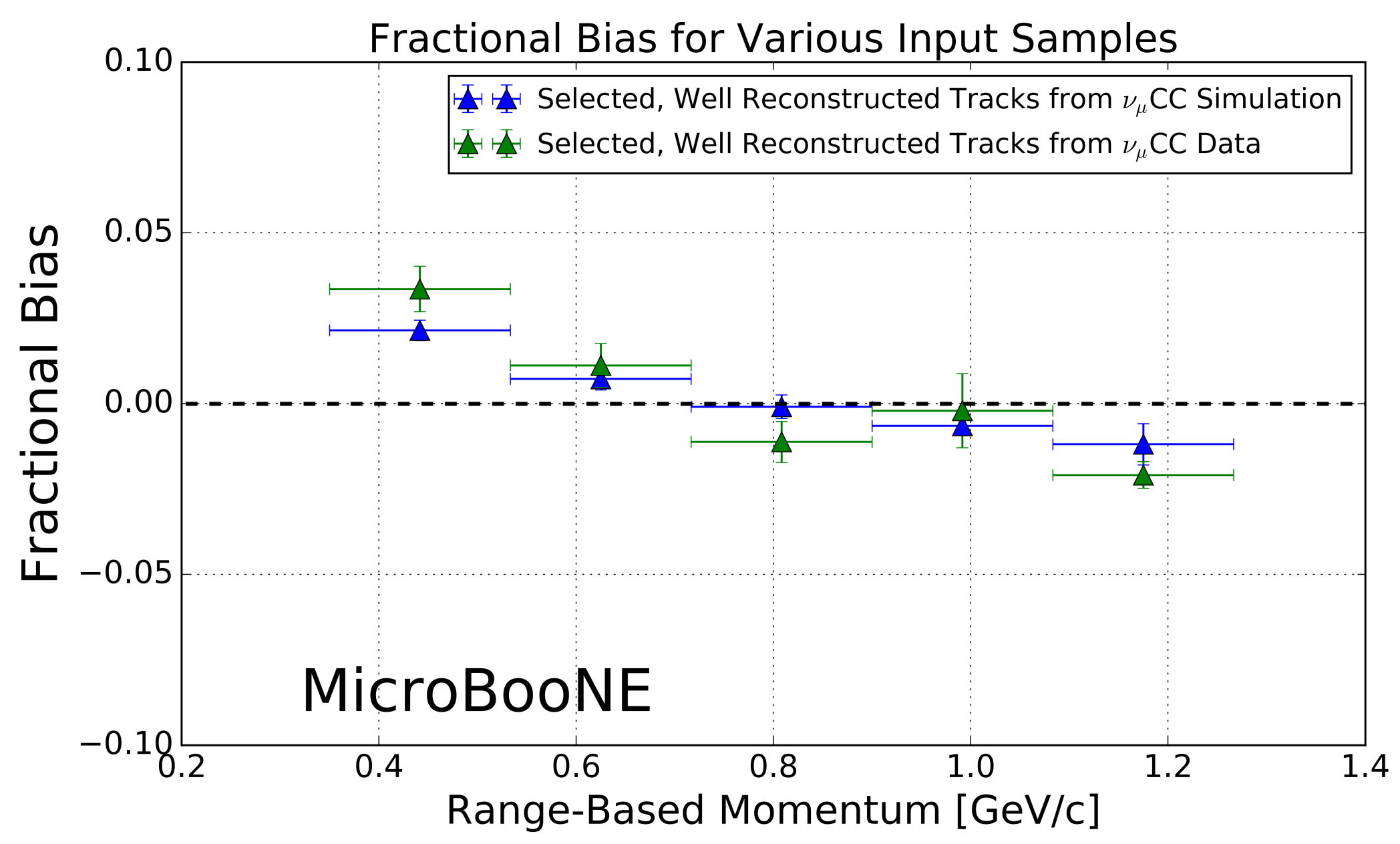




\section{MCS Performance on Contained Data Tracks}

- MCS resolution vs. range for both simulation and data

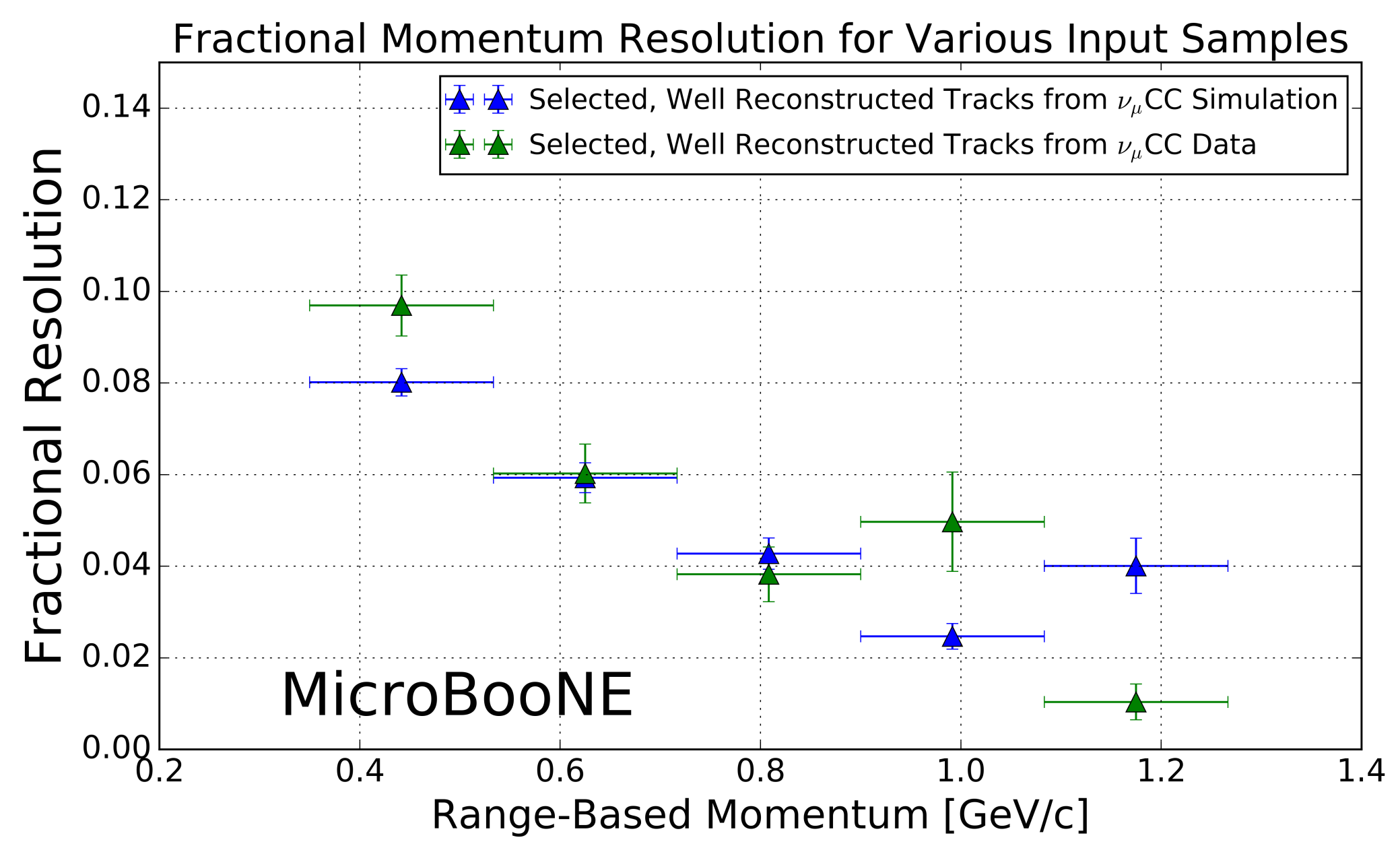


MCS Performance on Exiting Data Tracks 


\section{MCS Performance on Exiting Data Tracks}

- MCS will ultimately be used to determine the momentum of exiting muons in data, so it's very important to quantify this!

- But how would we measure this?

- Doesn't make sense to compare to momentum from range!

- Introduce "pseudo-exiting" tracks:

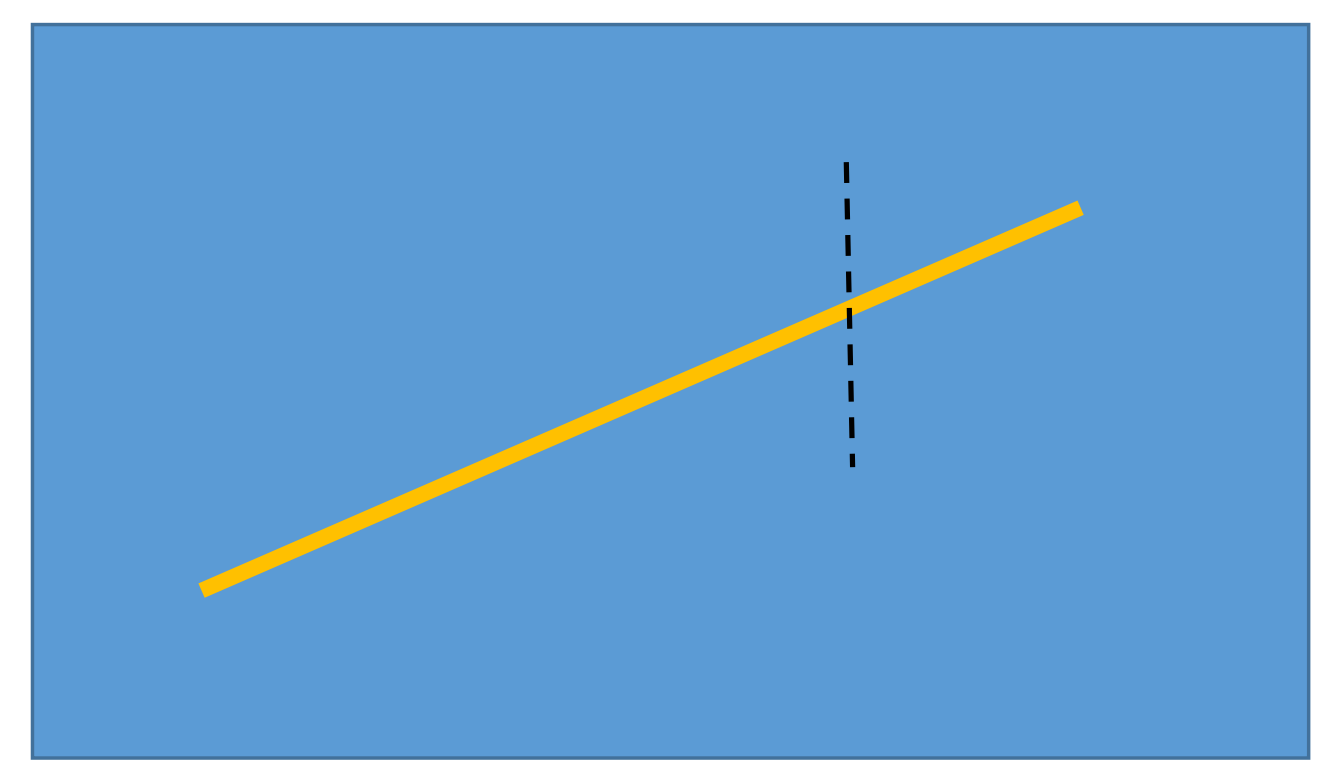

- Take a fully contained track, cut it off somewhere along its length

- We choose cutoff lengths of 2, 4, 6 segments exiting the fiducial volume (corresponding to $28 \mathrm{~cm}, 56 \mathrm{~cm}, 84 \mathrm{~cm}$, respectively)

- Note that we are limited in segment removal because we are dealing with contained tracks 


\section{MCS Performance on Exiting Data Tracks}

- First, we compared these pseudo-exiting tracks with real exiting tracks (all simulation)

- To do this, we placed a cut on length outside the TPC for real exiting tracks
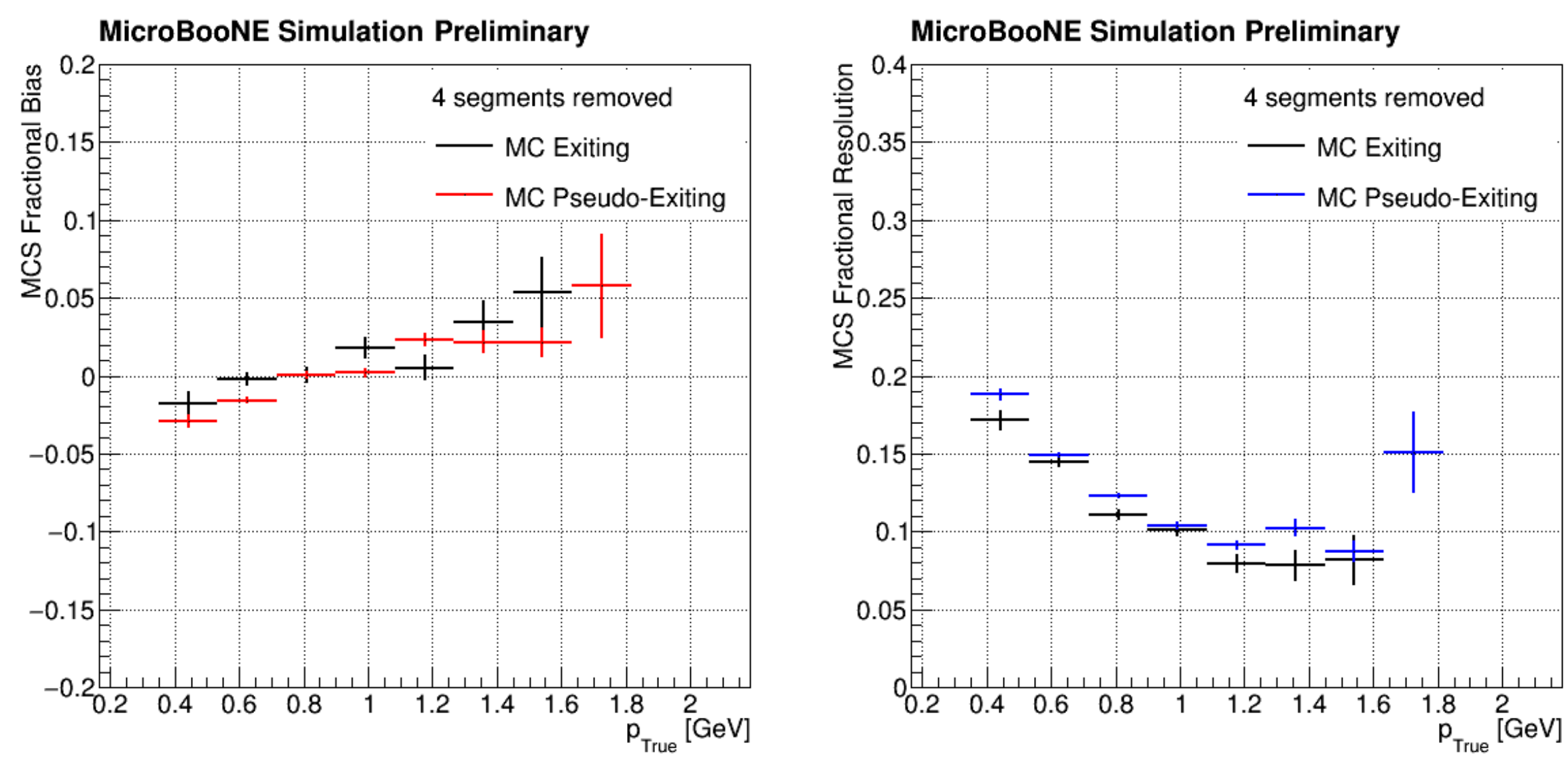


\section{MCS Performance on Exiting Data Tracks}

- Comparison of pseudo-exiting tracks for 2, 4, 6 segments removed

- MCS bias and resolution versus range
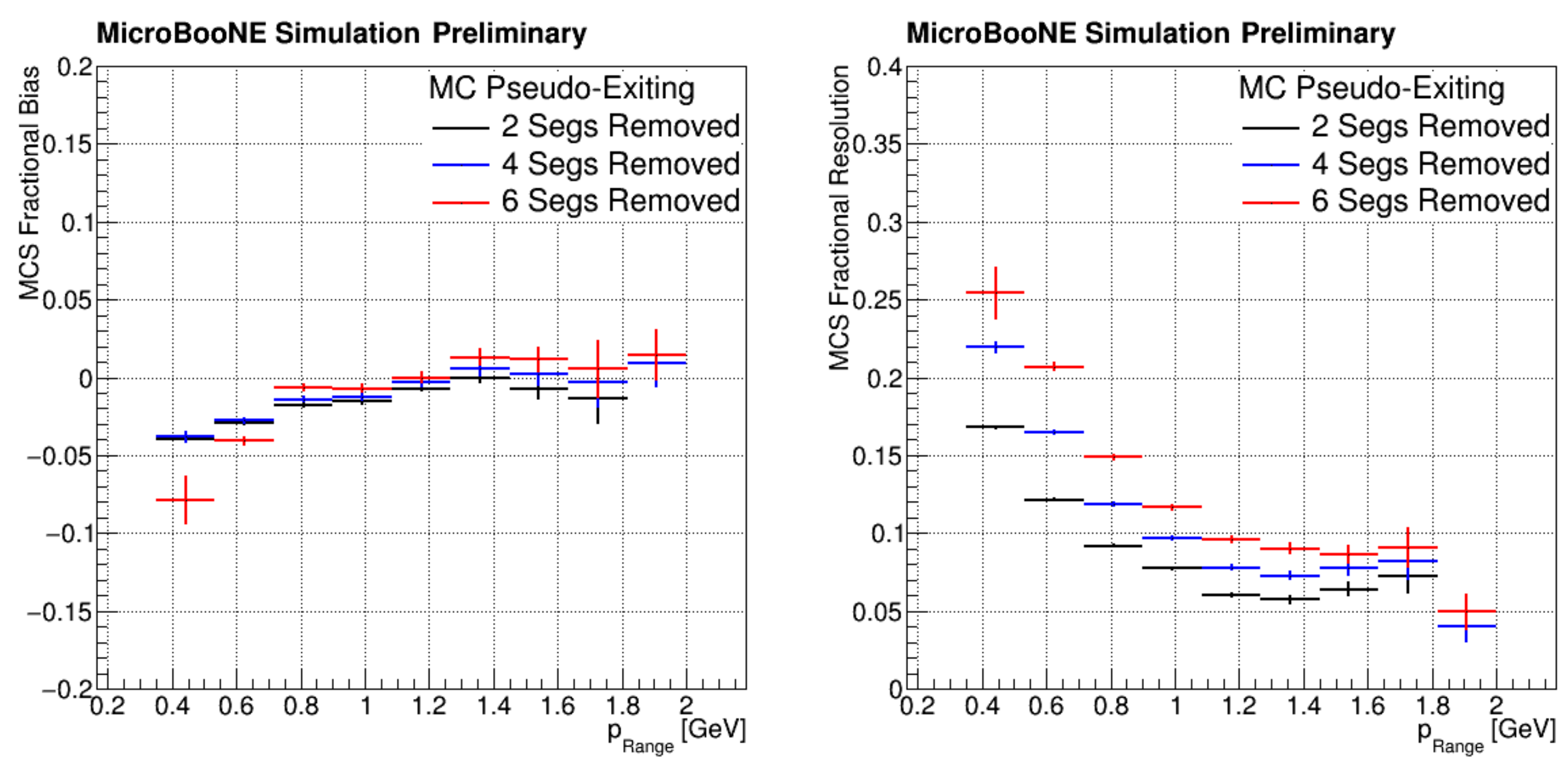


\section{MCS Performance on Exiting Data Tracks}

- Comparison of pseudo-exiting tracks and data (2 segments removed)

- MCS bias and resolution versus range
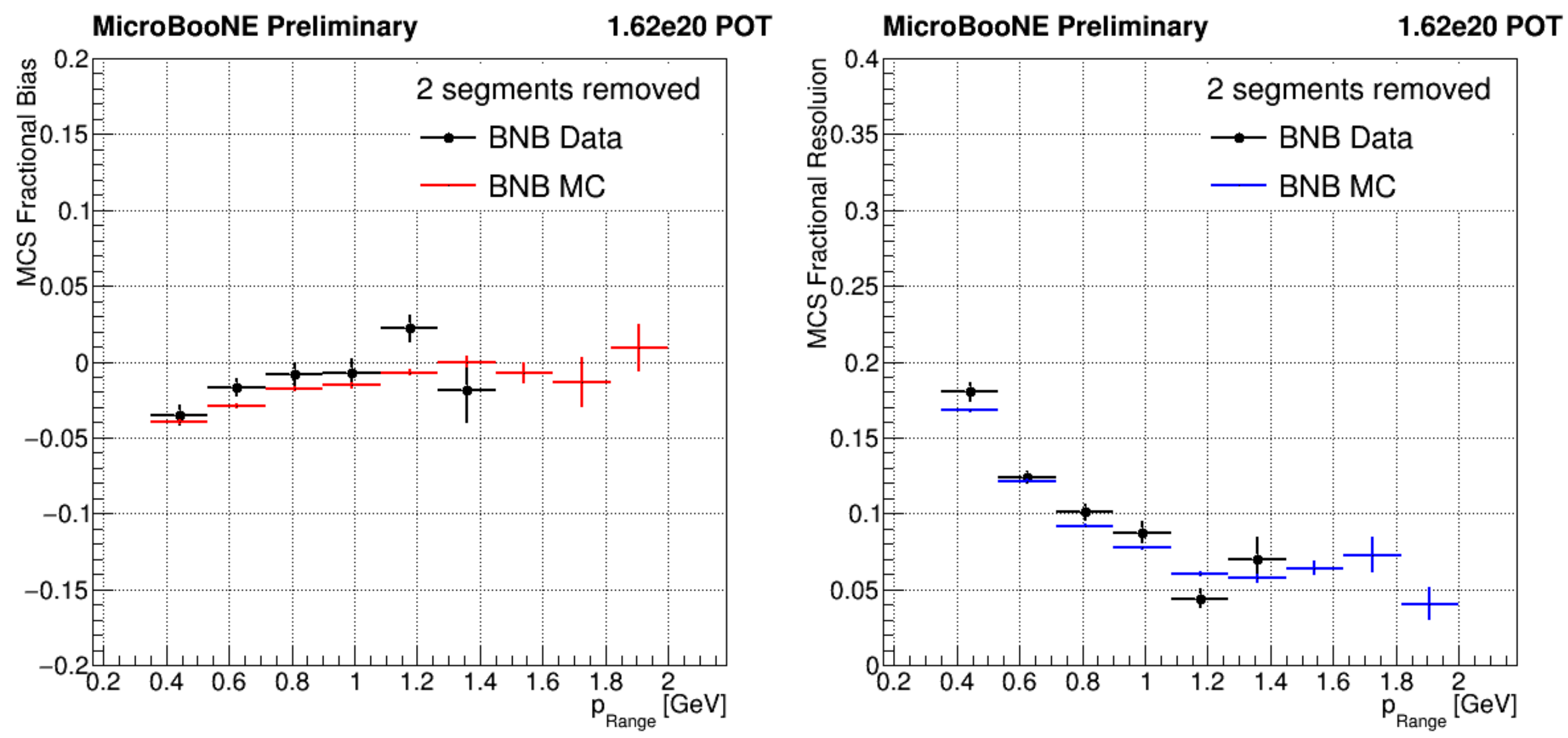


\section{MCS Performance on Exiting Data Tracks}

- Comparison of pseudo-exiting tracks and data resolution vs range

- 4, 6 segments removed
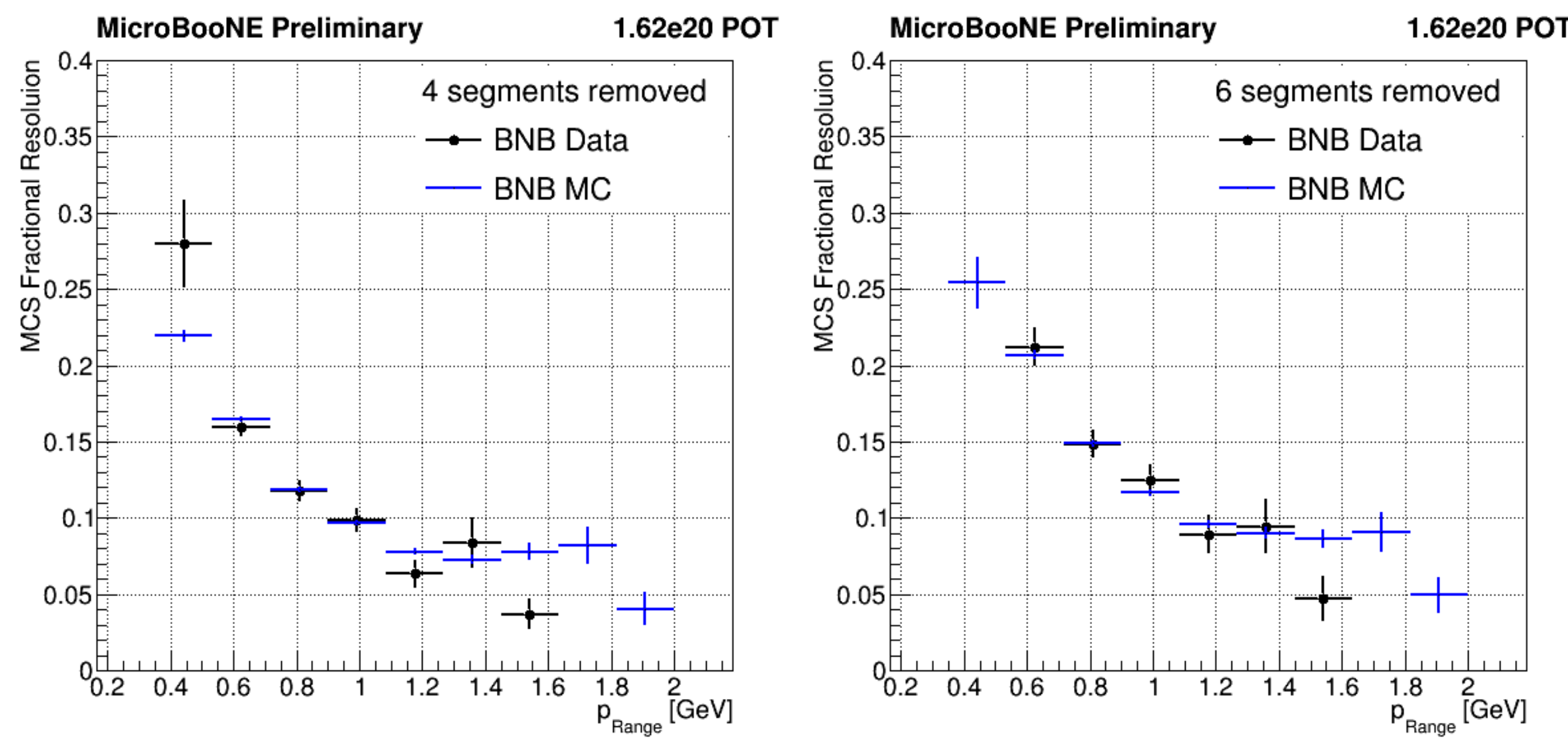


\section{Conclusion}

- Performance of MCS for contained tracks was shown to be within $5-10 \%$ in MicroBooNE data

- Comparable to the performance of MCS on contained simulated tracks

- Performance of MCS for exiting tracks in data has been shown to be within 5\% bias and under $20 \%$ resolution for 2 segments removed

- Under 30\% for 4, 6 segments removed

- Comparable to results from simulation

MicroBooNE is able to reconstruct the momentum of TPC-exiting

muons using MCS at a resolution of under $20-30 \%$ 


\section{Backup}




\section{The MicroBooNE Experiment}

- Part of the Short Baseline Neutrino (SBN) program at Fermilab

- Detector located 470 m from Booster Neutrino Beam (BNB) target

- Liquid Argon Time Projection Chamber (LArTPC) technology

- 90 tons active LAr in TPC (170 tons total in cryostat)

- Current largest TPC in the U.S. actively taking data
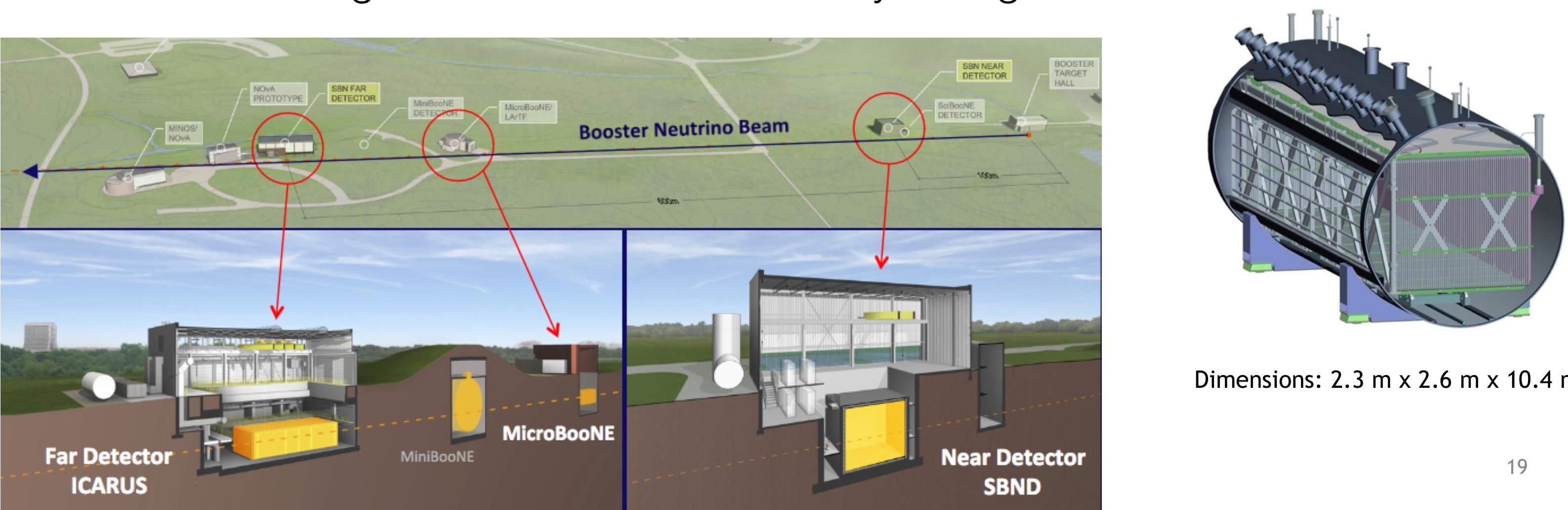

Dimensions: $2.3 \mathrm{~m} \times 2.6 \mathrm{~m} \times 10.4 \mathrm{~m}$ 
- Example distribution of fractional inverse momentum difference with the fit used to compute the bias (mean) and resolution (width) of the MCS method

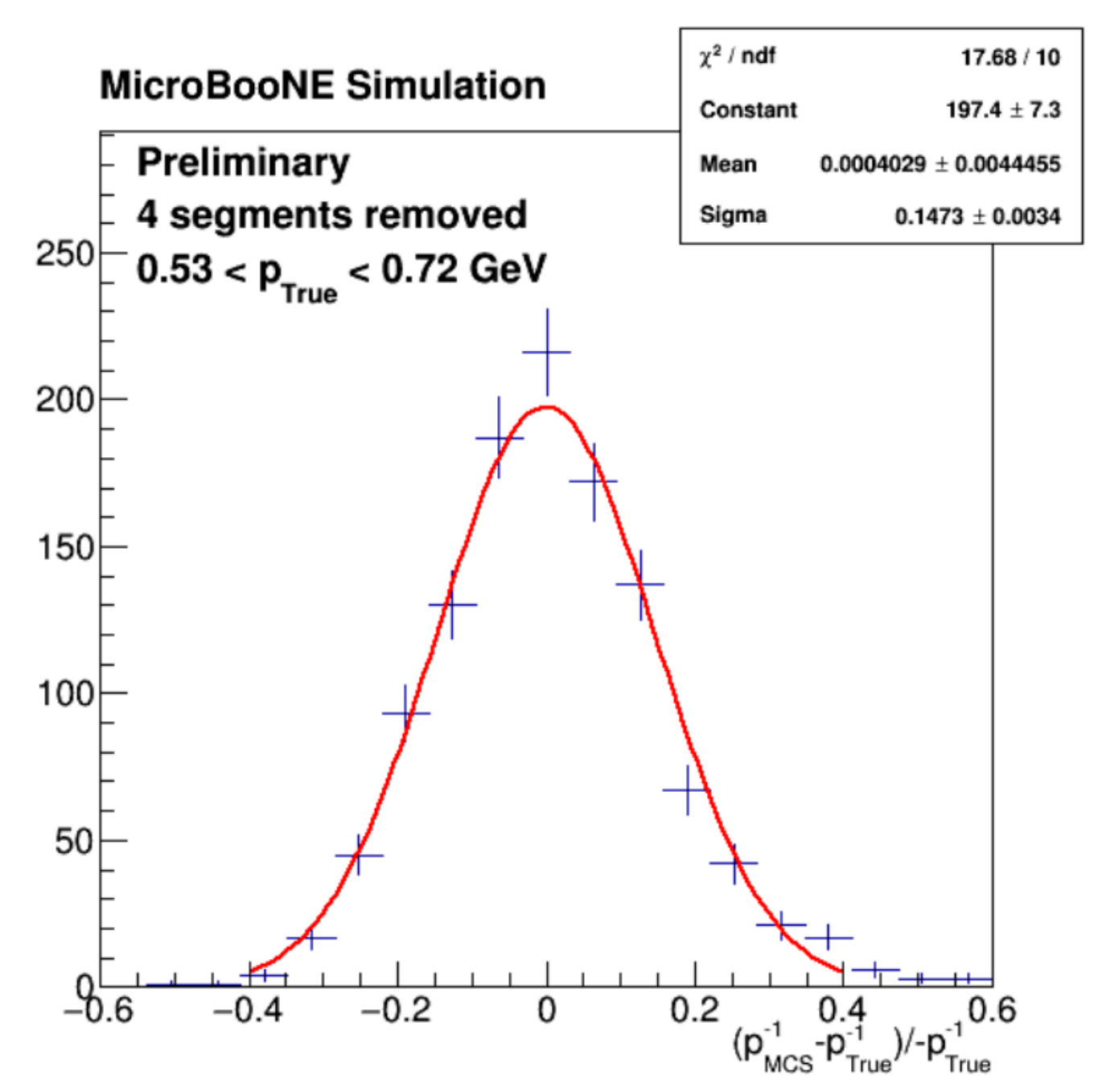




\section{Varying Segment Length}

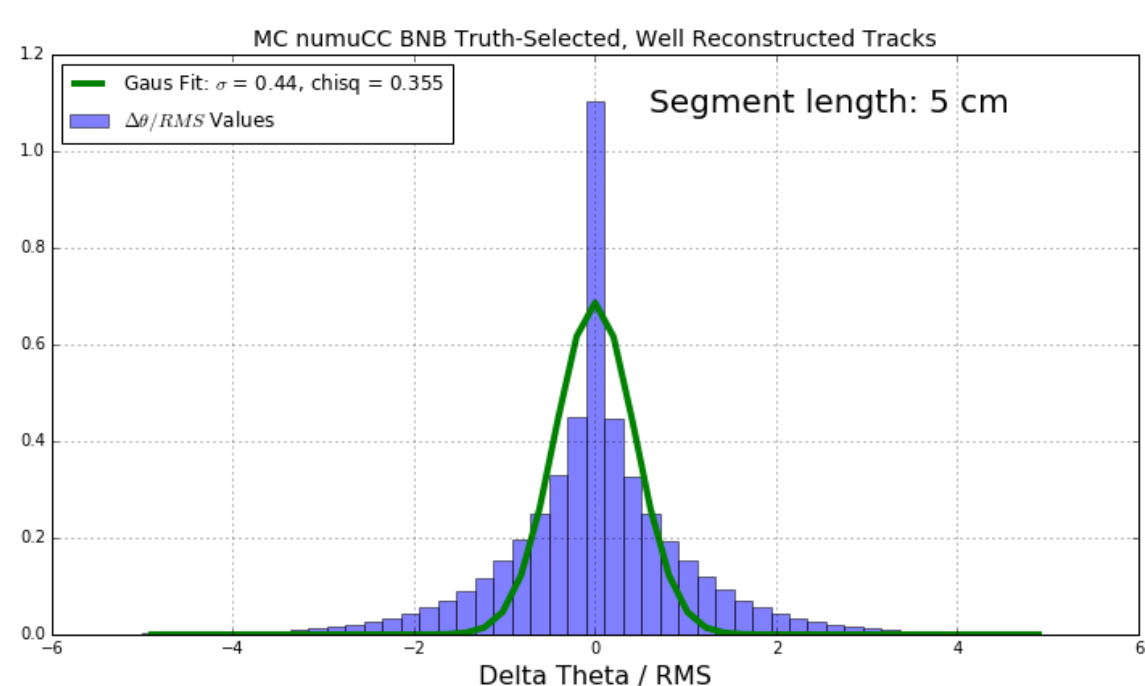

Delta Theta / RMS

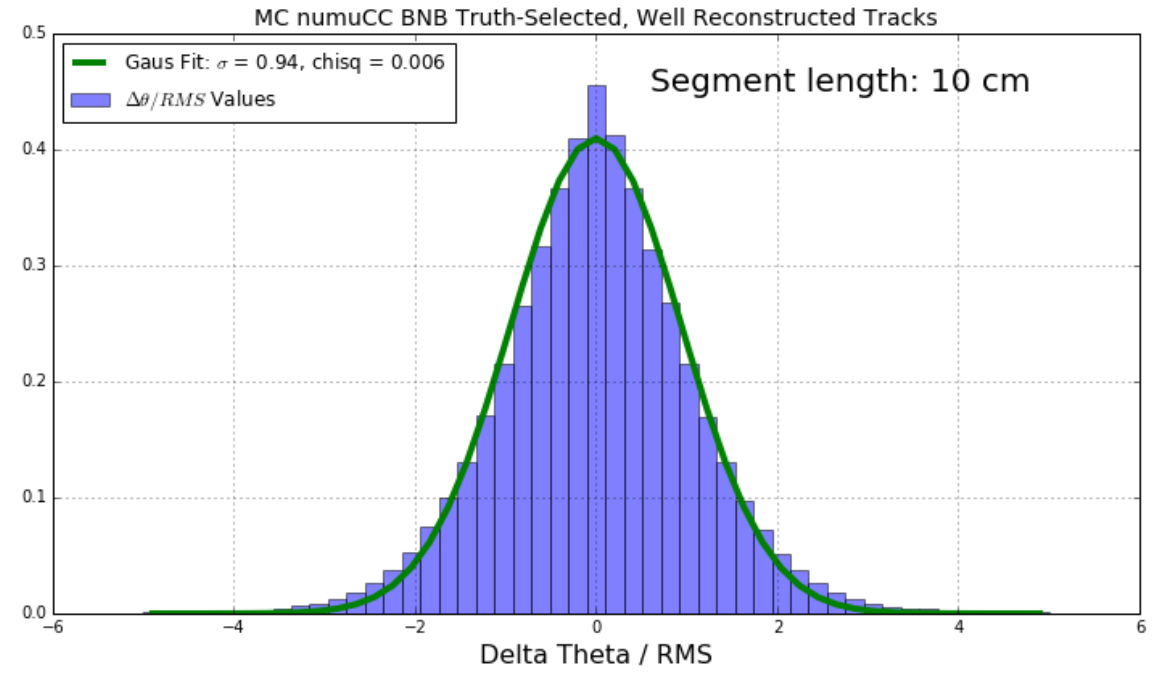

(b) Highland validation figure for $10 \mathrm{~cm}$ segment lengths.

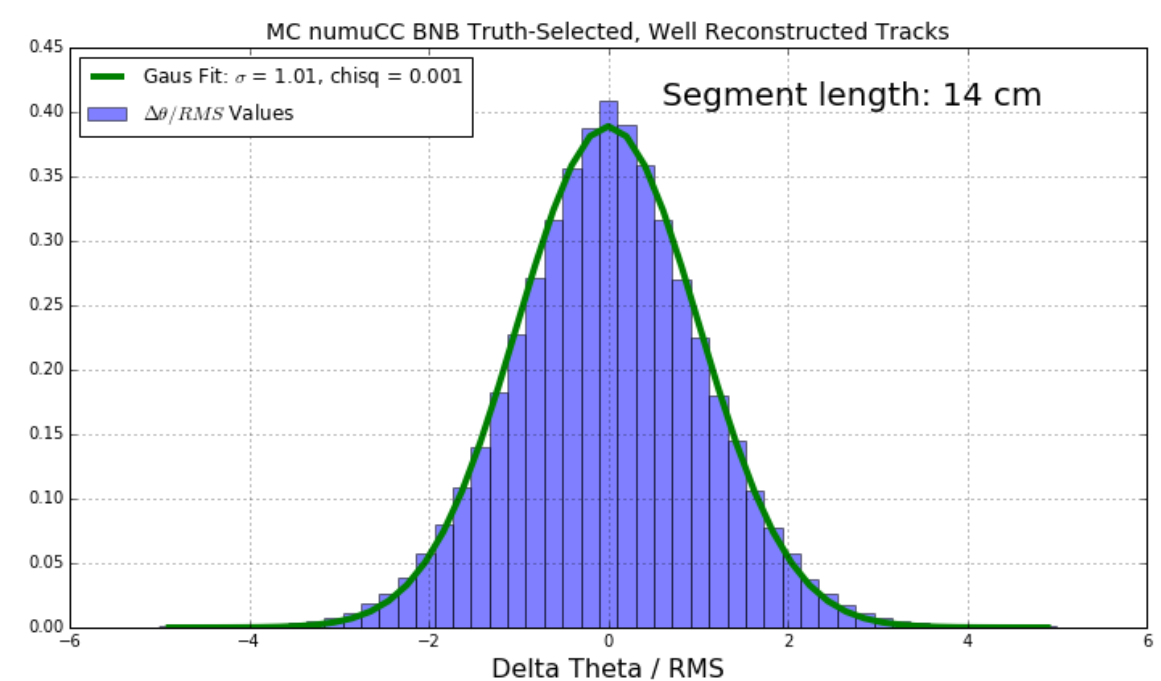

(c) Highland validation figure for $14 \mathrm{~cm}$ segment lengths.

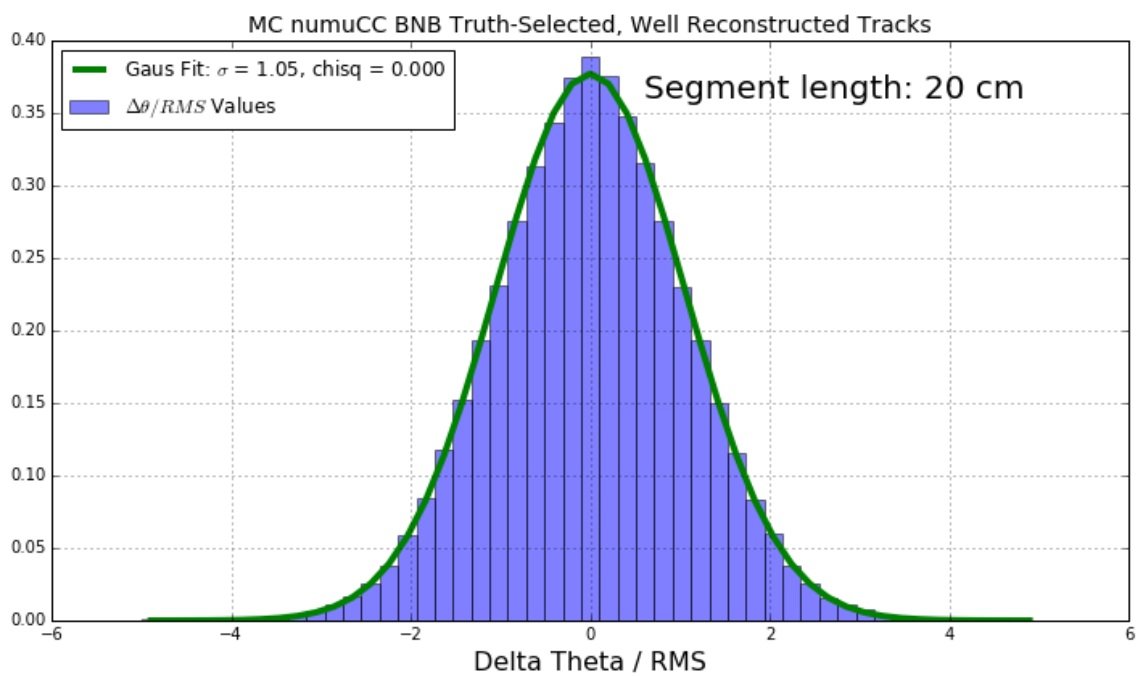

(d) Highland validation figure for $20 \mathrm{~cm}$ segment lengths. 
Why do we place a cut on $100 \mathrm{~cm}$ ?

Contained Tracks: Standard Deviation vs. Reco Length

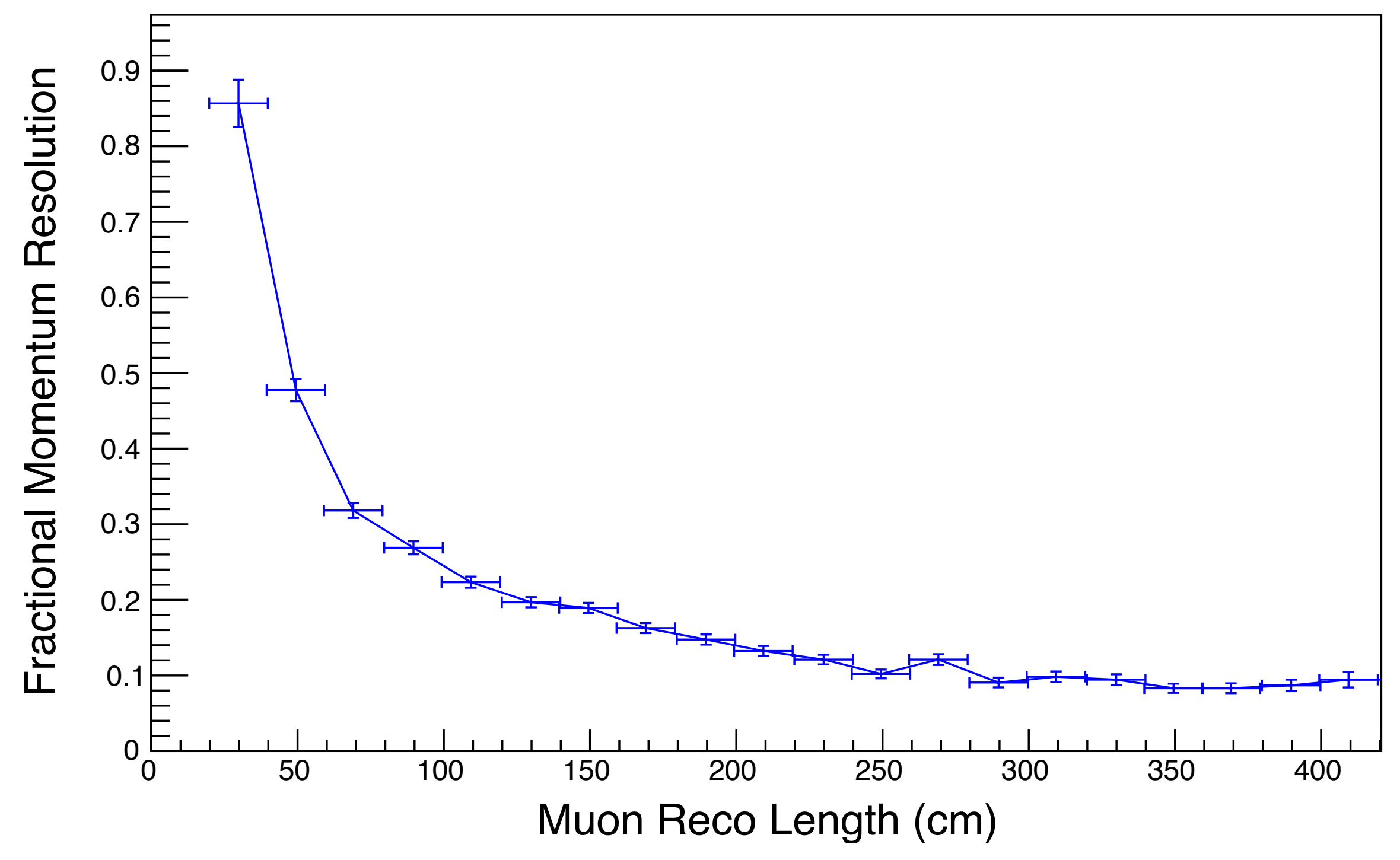

